\title{
Probabilistic Modeling of Space Shuttle Debris Impact
}

\author{
Luc J. Huyse, M. ASCE, Chris J. Waldhart, David S. Riha \\ Southwest Research Institute, San Antonio, Texas \\ luc.huyse@swri.org, cwaldhart@swri.org,driha@swri.org \\ Curtis E. Larsen \\ NASA Engineering and Safety Center, Langley Research Center, Hampton, Virginia \\ curtis.e.larsen@nasa.gov \\ Reynaldo J. Gomez, Phillip C. Stuart \\ NASA Johnson Space Center, Houston, Texas \\ reynaldo.j.gomez@nasa.gov,phillip.c.stuart@nasa.gov
}

\begin{abstract}
On Feb 1, 2003, the Shuttle Columbia was lost during its return to Earth. As a result of the conclusion that debris impact caused the damage to the left wing of the Columbia Space Shuttle Vehicle (SSV) during ascent, the Columbia Accident Investigation Board recommended that an assessment be performed of the debris environment experienced by the SSV during ascent. A flight rationale based on probabilistic assessment is used for the SSV return-to-flight. The assessment entails identifying all potential debris sources, their probable geometric and aerodynamic characteristics, and their potential for impacting and damaging critical Shuttle components.
\end{abstract}

A probabilistic analysis tool, based on the SwRI-developed NESSUS ${ }^{\circledR}$ probabilistic analysis software, predicts the probability of impact and damage to the space shuttle wing leading edge and thermal protection system components. Among other parameters, the likelihood of unacceptable damage depends on the time of release (Mach number of the orbiter) and the divot mass as well as the impact velocity and impact angle. A typical result is visualized in the figures below. Probability of impact and damage, as well as the sensitivities thereof with respect to the distribution assumptions, can be computed and visualized at each point on the orbiter or summarized per wing panel or tile zone.

\section{Introduction}

On Feb 1, 2003, the Shuttle Columbia was lost during its return to Earth. As a result of the conclusion that debris impact caused the damage to the left wing of the Columbia Space Shuttle Vehicle (SSV) during ascent, the Columbia Accident Investigation Board (CAIB, 2003) recommended that an assessment be performed of the debris environment experienced by the SSV during ascent. Eliminating the possibility of debris transport is not possible; therefore, a flight rationale based on probabilistic assessment is required for the SSV return-to-flight. The assessment entails identifying all potential debris sources, their probable geometric and aerodynamic characteristics, and their potential for impacting and damaging critical Shuttle components.

The analysis objective was to convert existing NASA analysis models and computer codes into an end-to-end probabilistic analysis tool for the assessment of external tank debris release, impact and damage to the orbiter (Huyse et al., 2006). Although the focus was mostly on the development of an analysis approach, a reliability assessment tool was 
built using the existing NESSUS code (NESSUS, 2006). NESSUS allows the user to perform probabilistic analysis with both analytical models and external computer programs such as NASA's debris transport codes.

\section{Probabilistic Modeling through Conditional Events}

The debris impact and damage mechanism are governed by the following events:

- E1: a piece of foam breaks off

- E2: debris travels down to and impacts the shuttle

- E3: impact is of sufficient force to damage shuttle beyond acceptable limit

In this probabilistic modeling a failure occurs if and only if these three events occur concurrently; mathematically this can be expressed as:

$$
\begin{aligned}
\operatorname{Pr}(\text { failure }) & =\operatorname{Pr}\left(E_{1} \cap E_{2} \cap E_{3}\right) \\
& =\operatorname{Pr}\left(E_{1}\right) \operatorname{Pr}\left(E_{2} \mid E_{1}\right) \operatorname{Pr}\left(E_{3} \mid E_{1} \cap E_{2}\right)
\end{aligned}
$$

This expression generally tracks the break down of the debris analysis into the several sub-disciplines involved: debris modeling, CFD analysis, impact modeling. Note that most of the discipline specific models are conditional in nature. For example, the CFD analysis used to predict the kinetic energy (event E2) of a divot inherently assumes that a divot has broken off $\left(E_{1}\right)$. In other words the CFD model does not so much model the event $E_{2}$ but the conditional event $E_{2} \mid E_{1}$.

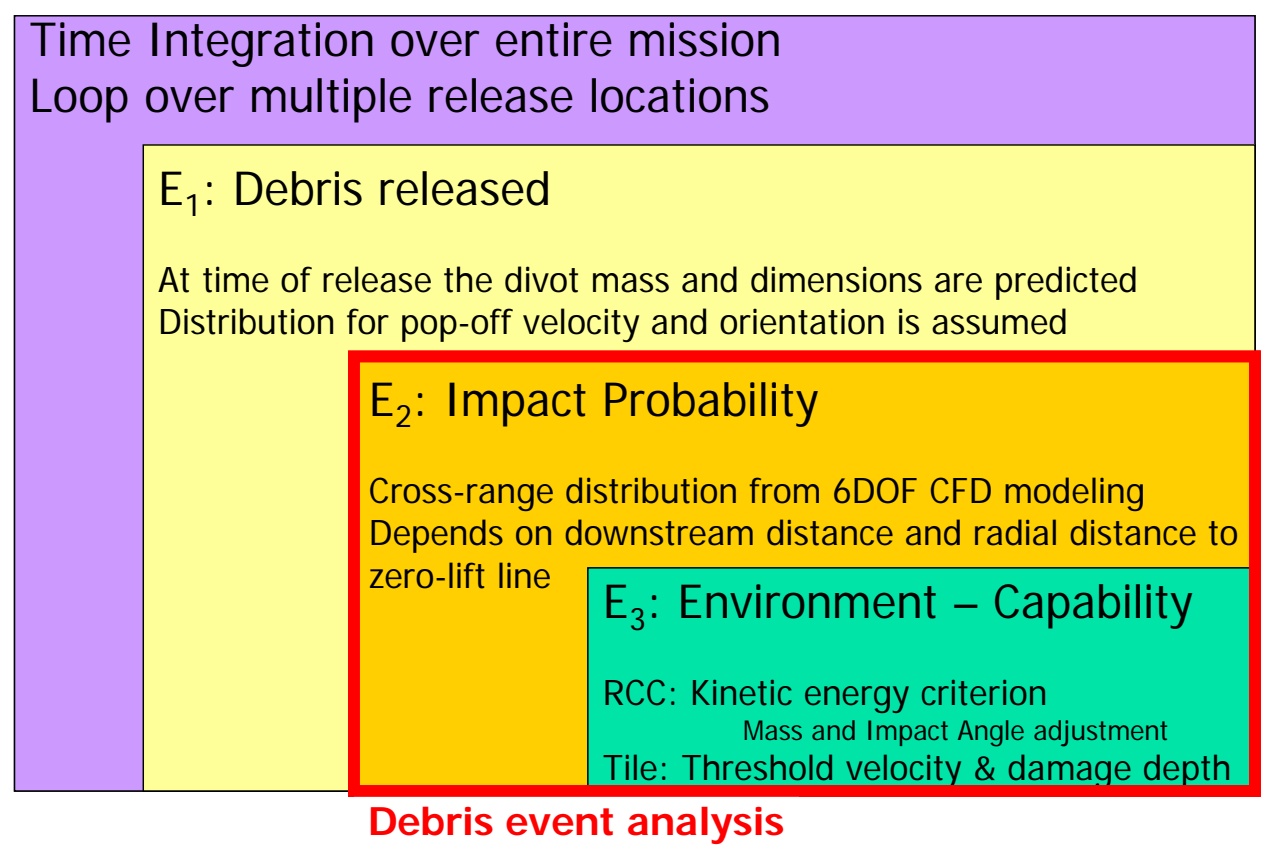

Figure 1: Functional outline of the probabilistic debris transport analysis 
Figure 1 describes how the conditional models are nested within each other due to the conditioning of each event upon the other events. It is worthwhile mentioning that the analysis of an in-flight debris release event, for which the release conditions are fairly well-known (from image analysis) can be achieved by considering only the events $\mathrm{E}_{2}$ and $\mathrm{E}_{3}$. In each of the subsequent sections the current state of the modeling will be described. The probability $\operatorname{Pr}\left(\mathrm{E}_{1} \cap \mathrm{E}_{2} \cap \mathrm{E}_{3}\right)$ gives the probability of unacceptable damage at a given time of release.

- Debris Release $E_{1}$ : time-dependent probability density functions were fitted to the ET debris tables generated by the Shuttle Program at the NASA Michoud Assembly Facility. A different release table must be created for each location of interest.

- Debris Transport and Impact $E_{2}$ : during transport, lift forces act to disperse the debris about their idealized, or zero-lift, trajectories. Therefore, the farther downstream the debris travels before impact, the greater the crossrange or dispersion. A rotationally symmetric cross-range distribution was modeled using 6DOF CFD results generated by NASA Ames and ELORET at Mach 2.5. Depending on the foam shape and initial rotation rate either a Weibull, lognormal or truncated normal distribution provides the best fit to the CFD results. This cross-range distribution is overlaid on top of the orbiter geometry to determine the probability of impact (Figure 2).

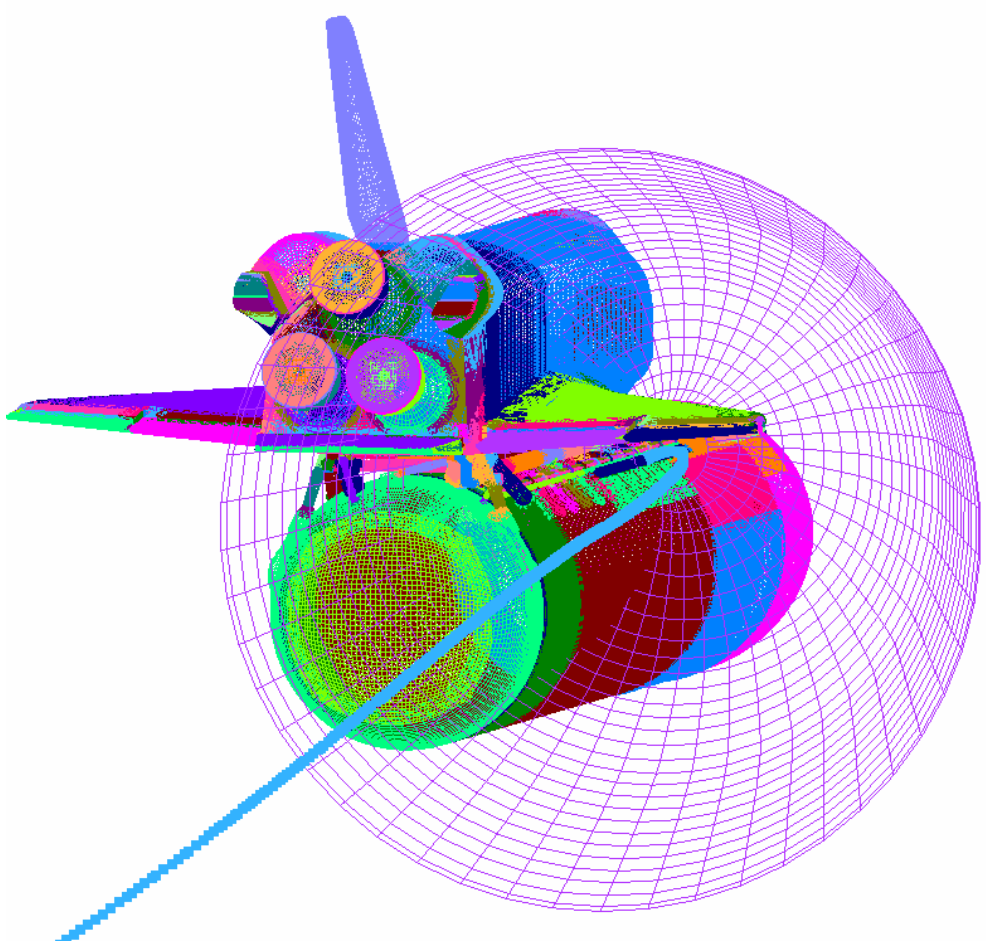

Figure 2: Zero-life life and 3-sigma cone for PAL ramp release location 
- Damage to Orbiter $E_{3}$ : a probabilistic capability model for both the reinforced carbon-carbon (RCC) wing leading edge and the orbiter tile has been implemented. A different distribution is used depending on the nature of the impact: two types of foam and ice are considered. A normal distribution is used for RCC and a Gumbel-min distribution is used for tile. The local incidence angle is an important driver for the RCC panel capability: the capability increases as the incidence angle becomes shallower.

\section{Uncertainty Modeling}

Various uncertainties affect the debris transport process. For instance, debris pieces travel downstream along a somewhat erratic path; advanced 6DOF CFD results were used to develop a stochastic model of this cross-range. Statistical descriptions of the RCC panel and tile zone capability were derived directly from experimental impact results. In addition to the highly stochastic nature of the release and transport of debris, uncertainties exist in the atmospheric conditions, mission profile, impact conditions, and material properties. Details of the uncertainty modeling can be found in Huyse et al., 2006.

The deterministic and probabilistic models were embedded in the NESSUS software and predict the probability of impact and damage to the space shuttle wing leading edge and thermal protection system components. A right-mouse click inside the problem definition window activates the Debris Transport Analysis (DTA) equation database (Figure 3). By simply clicking on the debris release location and time of interest, the appropriate model and variable definition is created.

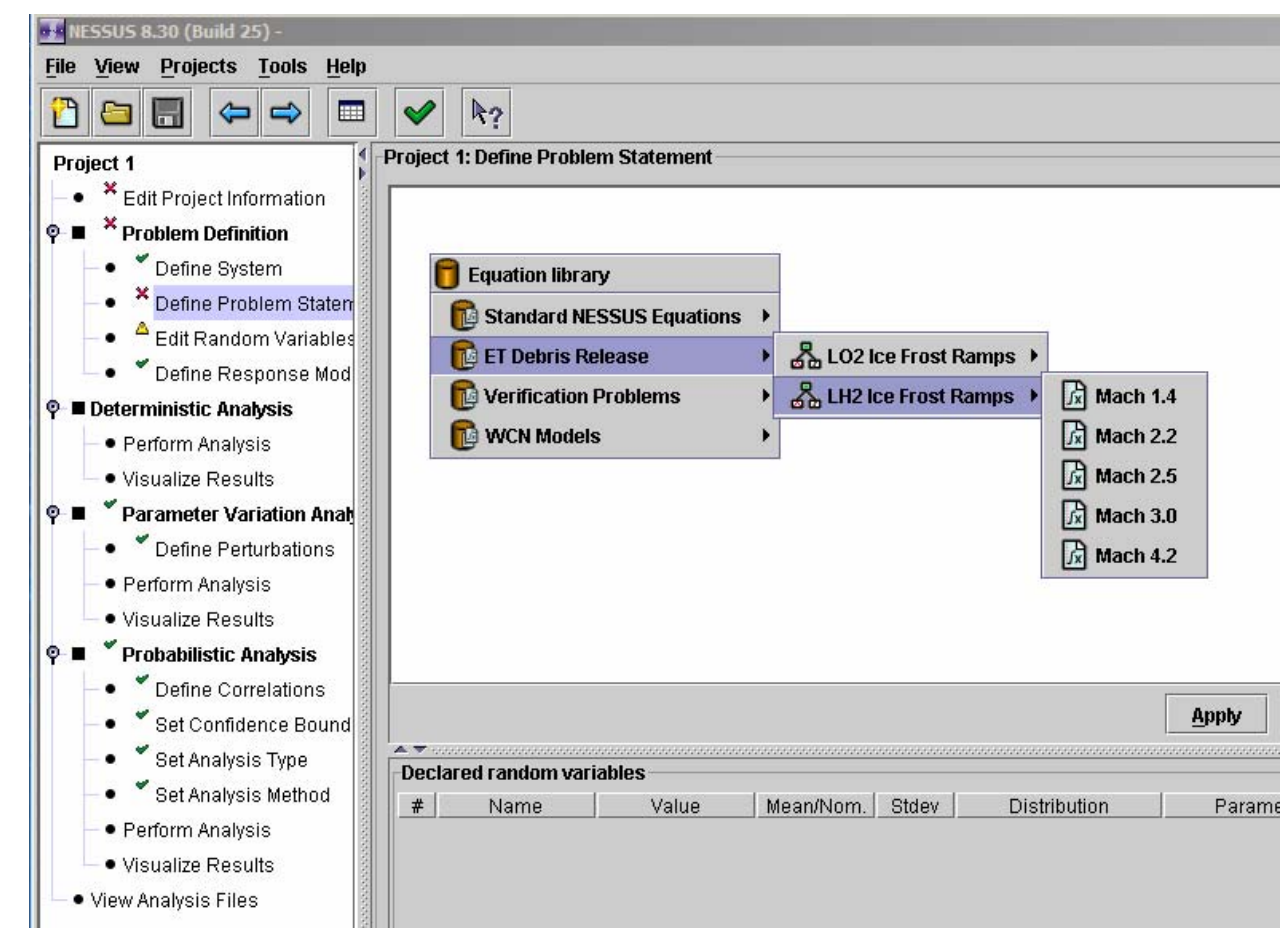

Figure 3: A right-mouse click in the NESSUS problem statement window activates the DTA equation database. 
The analysis tool is configured to enable quick analysis of any potential debris release event that may be recorded during the Orbiter's ascent into space. The conditional probabilistic analysis of a debris release can be performed very quickly (see Figure 4). For such an event the release conditions are - at least approximately - known and the probability $\operatorname{Pr}\left(E_{2} \cap E_{3} \mid E_{1}\right)$ is readily assessed.

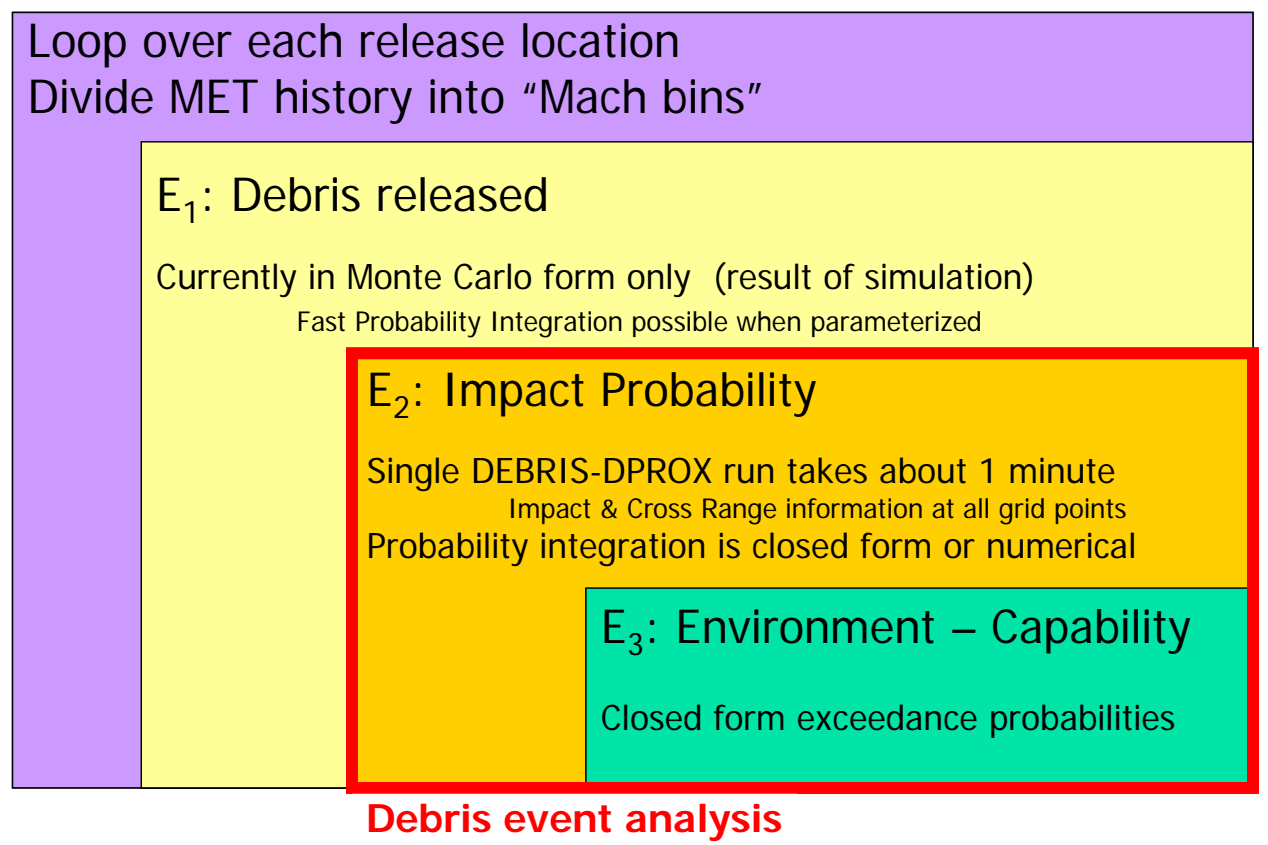

Figure 4: Overview of computational aspects in current debris transport model

In addition to the probability $\operatorname{Pr}\left(E_{2} \cap E_{3} \mid E_{1}\right)$, the code also generates plots of the sensitivity to a variety of distribution parameters such as the cross-range distribution. This is particularly of interest since the cross-range distribution type is dependent on an unknown initial rotation rate of the debris. These sensitivities point to the key drivers in the problem and were used to guide the allocation of further modeling and analysis efforts.

Among other parameters, the likelihood of unacceptable damage depends on the time of release (Mach number of the orbiter) and the divot mass as well as the impact velocity and impact angle. A typical result is visualized in Figure 5. Probability of impact and damage, as well as the sensitivities thereof with respect to the distribution assumptions, can be computed and visualized at each point on the orbiter or summarized per wing panel or tile zone. 

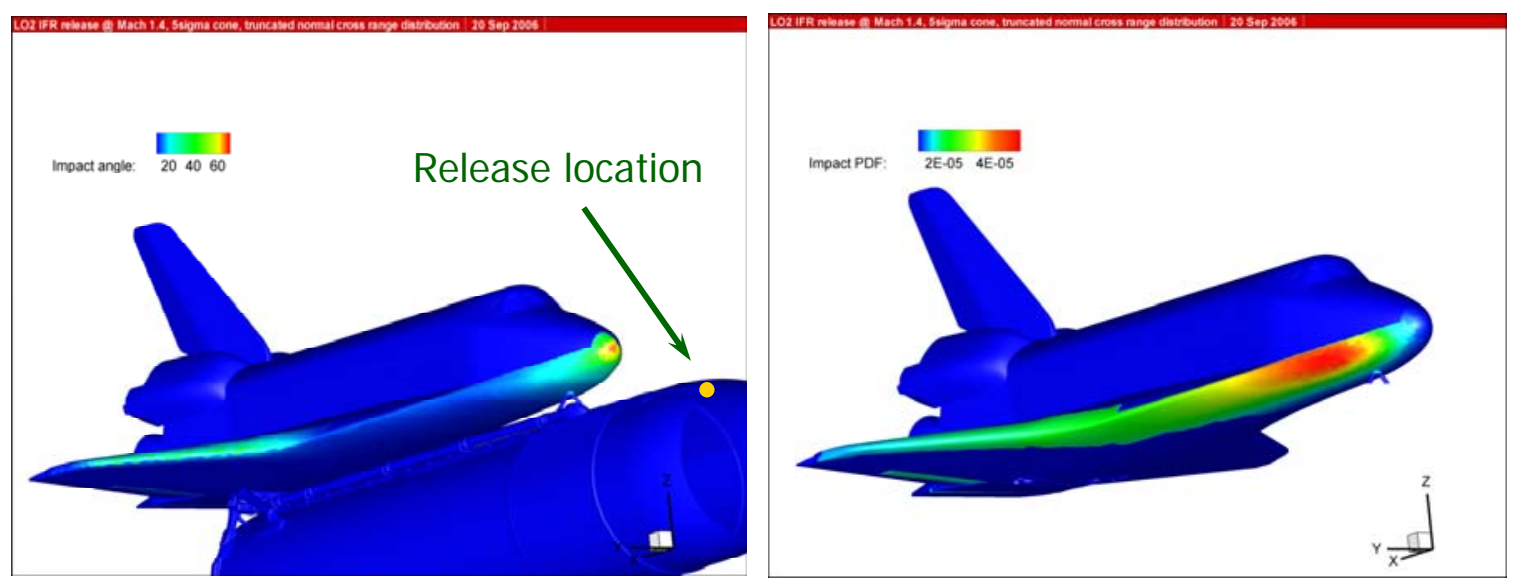

Figure 5. Typical result showing a possible release location and the impact angle (left) and probability of impact (right) to both the RCC panels and thermal protection shield on the Space Shuttle Orbiter.

\section{Summary and conclusions}

The probabilistic debris transport and damage analysis illustrates a practical approach to modeling conditional events. The probability of damage to the Shuttle is mitigated by including the conditions that the debris is released and that impact occurs. Accounting for all events in the sequence provides an accurate risk of failure. The probabilistic debris transport analysis specifically decoupled the release, transport, and damage events to evaluate either the probability of damage over the entire mission and orbiter, or to evaluate a specific event with a known release location, debris size, and Mach number.

\section{Acknowledgements}

The authors gratefully acknowledge funding provided by the NASA Engineering and Safety Center, made available under contract through Swales Aerospace (SwRI project 18.10592.03).

\section{References}

CAIB, (2003), Report of the Columbia Accident Investigation Board, Government Printing Office, Washington, DC. August, available online at: http://caib.nasa.gov/

Huyse, L.J., Waldhart, C.J., Riha, D.S., (2006), Shuttle Debris Probabilistic Analysis: Final Report and Recommendation to the NASA Engineering Safety Center, Southwest Research Institute, San Antonio, TX, October.

NESSUS, (2006), NESSUS User's Guide, v.8.4, Southwest Research Institute, San Antonio, TX. 